\title{
Supersymmetric quantum mechanics living on topologically nontrivial Riemann surfaces
}

\author{
Miloslav Znojil \\ Nuclear Physics Institute ASCR, \\ 25068 Řež, \\ Czech Republic* \\ Vít Jakubský \\ Departamento de Física, \\ Universidad de Santiago de Chile, \\ Casilla 307, Santiago 2, Chile $^{\dagger}$
}

May 30, 2022

*e-mail: znojil@ujf.cas.cz

${ }^{\dagger}$ e-mail: jakub@ujf.cas.cz 


\begin{abstract}
Supersymmetric quantum mechanics is constructed in a new non-Hermitian representation. Firstly, the map between the partner operators $H^{( \pm)}$is chosen antilinear. Secondly, both these components of a super-Hamiltonian $\mathcal{H}$ are defined along certain topologically nontrivial complex curves $r^{( \pm)}(x)$ which spread over several Riemann sheets of the wave function. The non-uniqueness of our choice of the map $\mathcal{T}$ between "tobogganic" partner curves $r^{(+)}(x)$ and $r^{(-)}(x)$ is emphasized.
\end{abstract}

\title{
KEYWORDS
}

supersymmetry, Schrödinger equation, complexified coordinates

\section{PACS}

11.30.Pb, 03.65.Fd, 93.65.Db 


\section{Introduction and summary}

We intend to show how, on an overall background of quantum mechanics, one could interconnect the purely algebraic concept of supersymmetry (SUSY, cf., e.g., review [1]) with the more or less purely analytic concept of quantum toboggans (cf. refs. [2, 3, 4, 5, 6] or a compact review paper [7]).

The presentation of this material will be initiated by section 2 on SUSY, followed by another introductory section 3 on complexifications of coordinates in quantum mechanics. On this background our main message will be delivered in sections 4 and 5. We shall show how a new class of SUSY representations can be constructed via models where the "coordinates" are defined as certain multisheeted complex curves $r(s)$.

The mathematical core of our present message lies in a never published observation that whenever one tries to intruduce complexified partner coor-

dinate curves $r(s)=r^{( \pm)}(s)$ in SUSY context, a nontrivial ambiguity of this partnership arises during the transition from the family of non-tobogganic curves (defined inside a single Riemann sheet, i.e., in the mere cut complex plane, $\left.r(s)=r^{(0)} \in \mathbb{C}\right)$ to the more general family of the curves $r(s)$ which interconnect several Riemann sheets of wave functions $\psi(r)$ in question.

\section{Supersymmetric quantum mechanics}

In review paper [1] one can find a virtually exhaustive list of reasons for interest in the so called supersymmetric quantum mechanics (SUSY QM). This list starts by the theory and phenomenology of elementary particles where SUSY QM plays the role of a methodical guide towards our understanding of its various mathematical features $[8,9,10$. At the other end of the list one finds a 
very close relationship of SUSY QM to certain exactly solvable one-dimensional potentials $V_{0}(x)$ defined most often as not too complicated functions of a single real coordinate $x$.

From the purely mathematical point of view one can characterize the majority of applications of SUSY QM as purely algebraic constructions. One simply picks up two arbitrary linear operators $A_{0}$ and $B_{0}$ acting in $\mathbb{L}^{2}(\mathbb{R})$, say,

$$
A_{0}=-\frac{d}{d x}+W(x), \quad B_{0}=\frac{d}{d x}+W(x)
$$

where $W(x)$ is a not yet specified "superpotential" and where one introduces the following pair of the so called "supercharges" acting in $\mathbb{L}^{2}(\mathbb{R}) \bigoplus \mathbb{L}^{2}(\mathbb{R})$,

$$
\mathcal{Q}_{0}=\left[\begin{array}{cc}
0 & 0 \\
B_{0} & 0
\end{array}\right], \quad \tilde{\mathcal{Q}}_{0}=\left[\begin{array}{cc}
0 & A_{0} \\
0 & 0
\end{array}\right] .
$$

The set of the anticommutators of these two operators is then easily shown to read

$$
\{\mathcal{Q}, \tilde{\mathcal{Q}}\}=\mathcal{H}, \quad\{\mathcal{Q}, \mathcal{Q}\}=\{\tilde{\mathcal{Q}}, \tilde{\mathcal{Q}}\}=0
$$

where we dropped subscripts and where we just have to add the following definition of the so called supersymmetric Hamiltonian,

$$
\mathcal{H}=\mathcal{H}_{0}=\left(\begin{array}{cc}
A_{0} B_{0} & 0 \\
0 & B_{0} A_{0}
\end{array}\right) .
$$

It is virtually trivial to verify the validity of the commutation relation

$$
[\mathcal{H}, \mathcal{Q}]=[\mathcal{H}, \tilde{\mathcal{Q}}]=0
$$

New operators do not emerge so that the algebra is closed as one of the simplest examples of supersymmetric algebra generated by the two elementary (super)charges and single (super)Hamiltonian. 
In the majority of the current applications of SUSY QM one usually extracts the superpotential $W(x)$ from the most common one-dimensional Schrödinger equation

$$
-\frac{d^{2}}{d x^{2}} \Phi_{0}(x)+V_{0}(x) \Phi_{0}(x)=E_{0} \Phi_{0}(x), \quad x \in(-\infty, \infty)
$$

for a ground-state wave function $\Phi_{0}(x)$ possessing no nodal zeros. Eq. (6) is being re-read as an equivalent nonlinear differential equation of the first order,

$$
V_{0}(x)-E_{0}=-\frac{d}{d x} W(x)+W^{2}(x) .
$$

This equation is satisfied by the superpotential $W(x)$ which can be also defined by the following explicit formula,

$$
W(x)=-\frac{1}{\Phi_{0}(x)} \frac{d}{d x} \Phi_{0}(x) .
$$

Alternatively, with a given ground-state wave function $\Phi_{0}(x)$ the latter two equations can be perceived as an explicit definition of the difference or, if you wish, of the zero-energy potential $U_{0}(x):=V_{0}(x)-E_{0}$.

In ref. [1] we tried to analyze the consequences of a transfer of the SUSY QM recipe (based on eqs. (77) and (8)) to the class of potentials

$$
V(r)=V^{(B G)}(r)=-r^{4}+\mathcal{O}\left(r^{2}\right)
$$

characterized by the "asymptotically anomalous" behavior. In the present paper we shall extend the results of ref. [11] to systems called quantum toboggans [2, 3]. 


\section{Quantum mechanics using complex coor- dinates $r$}

In spite of an asymptotically repulsive character of the quartic potential (9) "with wrong sign", Buslaev and Grecchi proved the reality of the spectrum under certain assumptions [12]. Moreover, they also explained why this spectrum remains discrete and bounded below, i.e., in principle, observable. Several further reasons for a thorough interest in the Buslaev's and Grecchi's model $V^{(B G)}(r)$ may be found, e.g., in ref. [13].

In the language of physics one of the most unusual assumptions connected with the use of eq. (9) lies, incidentally, in the manifest loss of the observability of the corresponding "coordinate" $r$. Indeed, one is allowed to consider its various complexified versions

$$
r=r_{\varepsilon}(x)=x-i \varepsilon(x)
$$

where $x \in(-\infty, \infty)$ is merely a parameter and where the function $\varepsilon(x)$ has been chosen as an $x$-independent positive constant in ref. [12]). Other choices with asymptotically growing $\varepsilon(x)$ were studied, e.g., in [14].

On this background another family of models called quantum toboggans (QT, [2]) may briefly be characterized by the existence of a topologically nontrivial Riemann surface $\mathcal{S}$ on which one can define wave functions [say, $\Phi_{0}(x)$ of eq. (6) ] by analytic continuation. This means that the class of the non-QT curves $r(x)$ given by eq. (10) is being complemented by all the smooth QT curves $r^{(N)}(x)$ which spread over several, $N>1$ sheets of $\mathcal{S}$.

One of the simplest illustrative examples of such a QT curve is given by eq. Nr. (10) of ref. [4,

$$
r^{(N)}(x)=-\mathrm{i}[\mathrm{i}(x-\mathrm{i} \varepsilon)]^{2 N+1}, \quad x \in(-\infty, \infty) .
$$


This formula describes a toboggan-reminding spiral which may only be approximated by eq. (10) at not too large $x$. Globally this curve encircles the origin $N$-times. Thus, a nontrivial $N$-dependence of the spectrum of energies may be expected to emerge whenever one finds a branch point in $\Phi_{0}(r)$ at $r=0$.

In the same manner, the existence of several branch points in $\Phi_{0}(r)$ would imply that one might consider a broader family of curves $r_{(\varrho)}^{(N)}(x)$ where the symbol $\varrho$ should distinguish between their topologically non-equivalent versions (a small demo is now available showing some $r_{(\varrho)}^{(N)}(x)$ for two branch points [15]).

\section{Supersymmetric quantum mechanics us- ing complex coordinates $r$}

The manifest impossibility of a return to the real-coordinate limits $\varepsilon \rightarrow 0$ in eqs. (10) or (11) will be of particular interest in SUSY QM. For a typical asymptotic ground-state-like solution of Schrödinger equation with potential (9) one would get the following contradiction at $\varepsilon=0$,

$$
\Phi_{0}(r)=\Phi_{0}^{( \pm)}(r) \sim \exp \left( \pm \frac{i r^{3}}{3}\right) \notin L_{2}(-\infty, \infty), \quad \varepsilon=0
$$

Turning attention to $\varepsilon \neq 0$ we may follow the idea presented in ref. [11] and start from one of the following two initial or tentative choices of the wave function,

$$
\begin{aligned}
& \Phi_{0}(r)=\psi^{(-)}(x)=(x-i \varepsilon) \exp \left(-i \frac{(x-i \varepsilon)^{3}}{3}\right) \in L_{2}(-\infty, \infty), \\
& \Phi_{0}(r)=\psi^{(+)}(x)=\frac{1}{x+i \varepsilon} \exp \left(+i \frac{(x+i \varepsilon)^{3}}{3}\right) \in L_{2}(-\infty, \infty) .
\end{aligned}
$$


In the light of eq. (8) this postulate would lead to the following two alternative, tentative superpotentials

$$
W^{( \pm)}(x)=-\left[\frac{d}{d x} \psi^{( \pm)}(x)\right] / \psi^{( \pm)}(x)= \pm\left[\frac{1}{x \pm i \varepsilon}-i(x \pm i \varepsilon)^{2}\right] .
$$

Although the application of standard rules would lead to the respective potentials

$$
\begin{gathered}
V^{(-)}(x)=-4 i(x-i \varepsilon)-(x-i \varepsilon)^{4}, \\
V^{(+)}(x)=\frac{2}{(x+i \varepsilon)^{2}}-(x+i \varepsilon)^{4}
\end{gathered}
$$

one can immediately verify that these potentials are not related by the standard SUSY partnership [11]. In the latter reference it has been found that the above-constructed pair of candidates for SUSY-related superpotentials satisfies a modified relation

$$
\left[W^{(+)}\right]^{2}-\left[\frac{d}{d x} W^{(+)}\right]=\mathcal{T}\left\{\left[W^{(-)}\right]^{2}+\left[\frac{d}{d x} W^{(-)}\right]\right\} \mathcal{T} .
$$

The symbol $\mathcal{T}$ stands for an antilinear and involutive operator of the usual complex conjugation. In the light of this observation it has been found in [11] that at a constant and positive $\varepsilon \neq 0$ only the minus-superscripted superpotentials are to be employed. Using this knowledge one reveals that the standard SUSY QM formulism of section 2] is still applicable, provided only that we update definition (11) as follows,

$$
A=-\mathcal{T} \frac{d}{d x}+\mathcal{T} W^{(-)}(x), \quad B=\frac{d}{d x} \mathcal{T}+W^{(-)}(x) \mathcal{T}
$$

Although these operators become antilinear, their use still leads to the same algebraic consequences as above, giving not only the manifestly non-Hermitian SUSY Hamiltonian

$$
\mathcal{H}=\left[\begin{array}{cc}
H^{(-)} & 0 \\
0 & H^{(+)}
\end{array}\right]=\left[\begin{array}{cc}
B A & 0 \\
0 & A B
\end{array}\right]
$$


but also the corresponding supercharges in the current form

$$
\mathcal{Q}=\left[\begin{array}{ll}
0 & 0 \\
A & 0
\end{array}\right], \quad \tilde{\mathcal{Q}}=\left[\begin{array}{ll}
0 & B \\
0 & 0
\end{array}\right] .
$$

This was the key observation made in ref. [11]. Unfortunately, its immediate transfer to QT models leads to difficulties. Let us now show how one may get rid of them.

\section{$5 \quad$ SUSY in tobogganic models}

\subsection{An ambiguity of $\mathcal{T}$ in cut plane}

For toboggans the direct application of the method of section 4 fails because

the current use of the operator $\mathcal{T}$ of complex conjugation requires that the coordinates remain real,

$$
(\mathcal{T} \psi)(\vec{r}):=\psi^{*}(\vec{r}), \quad \vec{r} \in \mathbb{R}^{d} .
$$

Then the antilinear operator $\mathcal{T}$ still can be perceived as interrelating the elements of the Hilbert space of states $|\psi\rangle$ with their dual partners $\langle\psi|$.

Once we leave the real space of $\vec{r}$ and, for the sake of definiteness, once we turn attention just to the one-dimensional example (10) we usually define our Hilbert space as a space of quadratically integrable functions of variable $r$. In such a case the operator of complex conjugation gets merely slightly modified,

$$
(\mathcal{T} \psi)(r(x)):=\psi^{*}\left(r^{*}(x)\right)
$$

In this form, unfortunately, it moves, at least formally, our wave functions out of the Hilbert space in which we started working. 


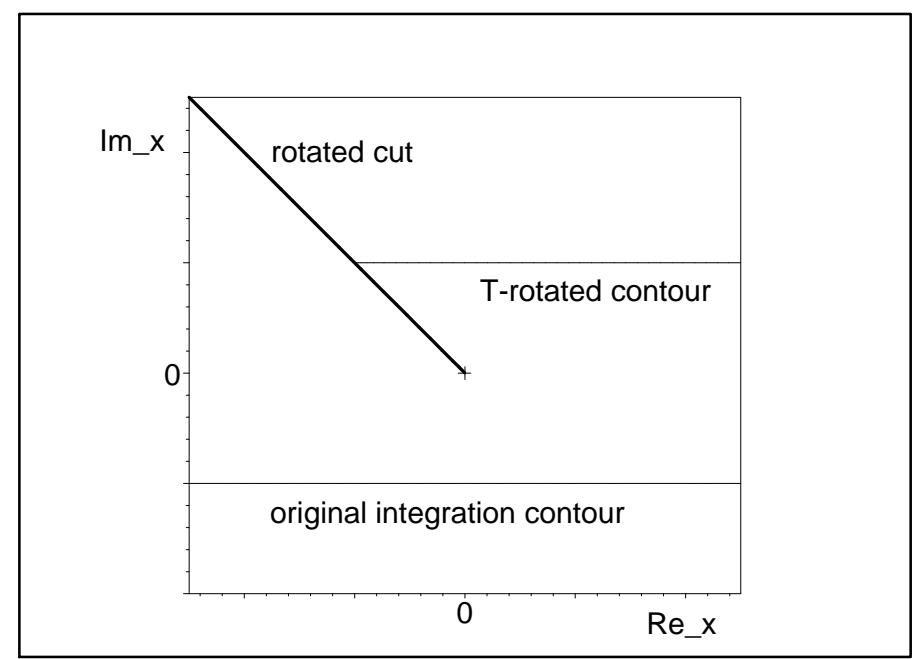

Figure 1: The complex line of coordinates (10) and its $\mathcal{T}$-transformed version with $\mathcal{T}=\mathcal{T}^{(+)}$. Our upwards-oriented cut is slightly rotated to sample a part of the next Riemann sheet.

The situation further worsens when your wave functions $\psi(r(x))$ become tractable as analytic (i.e., in principle, multivalued) functions of complex variable $r \in \mathbb{C}$. In Figure 1 we visualize the situation where the function $\psi(r)$ (defined along the complex contour (10) - see the lower horizontal line in the picture) is assumed to possess a branch point located, say, in the origin of the complex $r$-plane. Then, of course, we have to draw a cut from the origin (say, upwards) and restrict our attention just to the resulting part of the Riemann surface $\mathcal{S}$ of $\psi(r)$ (i.e., to its zeroth sheet $\mathcal{S}_{0}$ ). This enables us to "see" all the curves $r(x)$ as well as the functions $\psi[r(x)]$ which live on them $\}$. At the same time, this disables us to "see" all the curve $r^{*}(x)$. In order to "see" it \{and, of course, also the $\mathcal{T}$-image of our initial function $\psi[r(x)]\}$ we would have to move to one of the neighboring Riemann sheets (denoted, conveniently, as $\mathcal{S}_{-1}$ and $\left.\mathcal{S}_{+1}\right)$. 


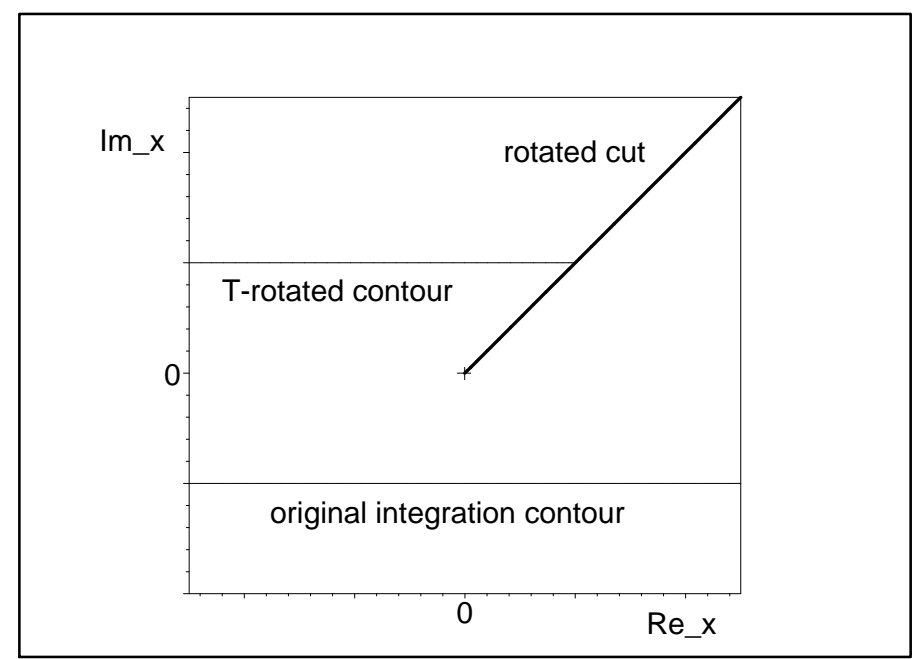

Figure 2: The complex line of coordinates (same as in Figure 1) and its $\mathcal{T}$-transformed version with $\mathcal{T}=\mathcal{T}^{(-)}$. The cut is now slightly rotated in opposite direction.

Schematically, these two possibilities are illustrated in Figures 1 and 2 where we see that in the presence of the branch point(s) in $\psi(r)$, the "complex conjugation" of the initial "curve of complex coordinates" $r(x)$ [exemplified by eq. (10)] can be mediated by at least two nonequivalent formal operators $\mathcal{T}^{( \pm)}$ where the superscript indicates our choice between the two eligible Riemann sheets $\mathcal{S}_{ \pm 1}$ to which we move. At the same time, it is also necessary to imagine that for the generic, logarithmic form of $\mathcal{S}$ our antilinear maps will also lose their involutive character so that $\left(\mathcal{T}^{( \pm)}\right)^{2} \neq I$ in general. 


\subsection{The problem of classification of families of $\mathcal{T} \mathrm{s}$ on Riemann surfaces}

All the above considerations show that we must be very careful with the notation conventions whenever a multivalued, analytic wave function lives on some

less trivial QT curve $r_{(\varrho)}^{(N)}(x)$. Once more we have to recollect the dichotomy between the choice of the operators $\mathcal{T}=\mathcal{T}^{(+)}$or $\mathcal{T}=\mathcal{T}^{(-)}$as encountered under assumption that there exists just a single branch point in $\mathcal{S}=\mathcal{S}^{[1]}$ (cf. our Figures 1 and 2).

One immediately imagines that for all the Riemann surfaces $\mathcal{S}=\mathcal{S}^{[M]}$ which are "punctured" by more (i.e., in general, $M \geq 1$ ) branch points the classification of all the eligible QT paths $r(x)$ gets quickly very complicated. In particular, the dichotomy illustrated by our Figures 1 and 2 must be understood locally at $M>1$. The above-mentioned $M=1$ distinction between $\mathcal{T}=\mathcal{T}^{(+)}$and $\mathcal{T}=\mathcal{T}^{(-)}$must be performed in the vicinity of every branch point $r_{B P}^{[K]}$ with $K=1,2, \ldots, M$. In this way a superscripted $M$-component multiindex $\varrho=( \pm, \pm, \ldots, \pm)$ appears also in every specific choice of the generalized complex conjugation,

$$
\mathcal{T}=\mathcal{T}^{(\varrho)} \neq \mathcal{T}^{-1}
$$

Marginally let us note that a set of nice analytic examples of wave functions where $M$ went up to five has been constructed by Sinha and Roy [16] and that the amazing combinatorial complications related to an exhaustive classification of the non-equivalent QT paths already emerge in the first less trivial case with $M=2[5]$.

This being said, our final return to the QT SUSY QM is very easy because once we overcome the combinatorial classification barriers and choose any particular member $\mathcal{T}$ of the family of conjugations (22) we only have to modify 
our above-mentioned formula (17) and set

$$
A=-\mathcal{T} \frac{d}{d x}+\mathcal{T} W^{(-)}(x), \quad B=\frac{d}{d x} \mathcal{T}^{-1}+W^{(-)}(x) \mathcal{T}^{-1} .
$$

In the purely algebraic language, all the rest of the construction of the general QT-type SUSY generators remains fully analogous to the above-described recipe. In contrast, in the language of analytic functions the situation seems much more exciting due to the flexibility of the resulting variability of the paths of coordinates $r(x)$. At the same time, in is worth adding that any more quantitative evaluation (say, of the related numerical spectra of energies) still seems to be a fairly difficult open question at present [6].

\section{Acknowledgements}

MZ acknowledges Universidad de Santiago de Chile for hospitality and GAČR grant Nr. 202/07/1307 and MŠMT “Doppler Institute” project Nr. LC06002 for supplementary support. VJ was supported by FONDECYT under grant 3085013. Collaboration also partially supported by DICYT (USACH).

\section{References}

[1] F. Cooper, A. Khare and U. Sukhatme, Phys. Rep. 251, 267 (1995).

[2] M. Znojil, Phys. Lett. A 342, 36 (2005).

[3] M. Znojil, J. Phys. A: Math. Gen. 39, 13325 (2006);

M. Znojil, Phys. Lett. A 372, 3591 (2008).

[4] M. Znojil, J. Phys. A: Math. Theor. 41 (2008) 215304.

[5] M. Znojil, Phys. Lett. A 372, 584 (2008). 
[6] G. J. C. Wessels, A numerical and analytical investigation into nonHermitian Hamiltonians (University of Stellenbosch, 2008, Master-degree thesis supervised by H. B. Geyer and J. A. C. Weideman).

[7] M. Znojil, J. Phys.: Conference Series 128, 012046 (2008).

[8] Y. A. Gelfand and E. P. Likhtman, JETP Lett. 13, 323 (1971);

P. Ramond, Phys. Rev. D 3, 2415 (1971);

A. Neveu and J. Schwarz, Nucl. Phys. B 31, 86 (1971).

[9] E. Witten, Nucl. Phys. B 188, 513 (1981).

[10] E. Witten, Nucl. Phys. B 202, 253 (1982).

[11] M. Znojil, F. Cannata, B. Bagchi and R. Roychoudhury, Phys. Lett. B $483,284(2000)$.

[12] V. Buslaev and V. Grecchi, J. Phys. A: Math. Gen. 26, 5541 (1993).

[13] C. M. Bender, D. C. Brody, J.-H. Chen, H. F. Jones, K. A. Milton, M. C. Ogilvie, Phys. Rev. D 74, 025016 (2006).

[14] H. F. Jones and J. Mateo, Phys. Rev. D 73, 085002 (2006); C. M. Bender and D, W. Hook, J. Phys. A: Math, Theor. 41, 244005 (2008).

[15] Jan Novotný, http://demonstrations.wolfram.com/TheQuantumTobogganicPaths

[16] A. Sinha and P. Roy, Czechosl. J. Phys. 54, 129 (2004). 